\title{
MASTER
}

NEUTRONS SCATTERING STUDIES OF THE ACTINIDES

by

G. H. Lander

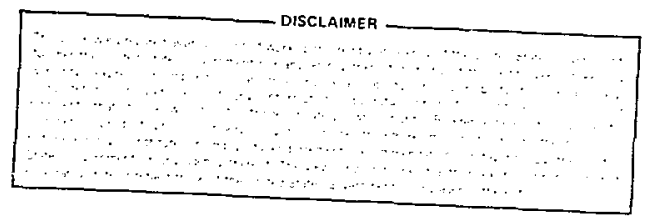

Prepared for

International Magnetism Conference

Munich, Germany

September 3-7, 1979

ARGONNE NATIONAL LABORATORY, ARGONNE, ILLINOIS

Operated under Contract W-31-109-Eng-38 for the

U. S. DEPARTMENT OF ENERGY 
NEUTRON SCATIERING STUDIES OF THE ACTINIDES

G. H. Lander

Argonne National Laboratory*, Argonne, IL 60439 U.S.A.

\begin{abstract}
The electronic structure of actinide materials presents a unique example of the interplay between localized and hand electrons. Together with a variety of other techniques, especially magnetization and the Mossbaver effect, neutron studies have helped us to understand the systcmatics of many actinide compounds that order magnetically. A direct consequence of the localization of $5 f$ electrons is the spin-orbit coupling and subsequent spin-lattice interaction that often leads to strongly anisotropic behavior. The unusual phase transition in $\mathrm{UO}_{2}$, for example, arises from interactions between quadrupole moments. On the other hand, in the moropnictides and monochalcogenides, the anisotropy is more difficult to understand, but probably involves an interaction between actinide and anion wave functions. A variety of neutron experiments, including formfactor studies, critical scattering and measurements of the elementary excitations have now been performed, and the conceptual picture emerging from these studies will be discussed.
\end{abstract}

\title{
Introduction
}

The most important single factor giving rise to the varied properties. of actinldes is the extended nature of the $5 f$ electron wave functions. As a consequence of their spatial extent, the $5 f$ electrons play a much greater role in bonding and related properties than the $4 f$ electrons in the lanthanide series. At the same time the $5 f$ electrons have an orbital component, $\ell=3$, 
that provides a potentlal for localization and such phenomena as a strong spin-lattice interaction. We might, therefore, expect properties ranging from that of itinerant $3 d$ systens to that of localized $4 f$ systems and, indeed, the literature of the actinides bears witness to a rich variety of magnetic belhavior [1]. The new physics, however, seens likely to emerge from the examples of behavior intermediate between those of the other two major magnetic series, where existing theories can be tested in their limits.

In discussing experiments on transuranium materials everybody is aware, of course, of both the radioactivity and toxicity. In addition, large quantities of samples are seldom available, and neutron investigations are therefore at a disadvantage, since they often require at least 1 gram, and, for complex experiments involving inelastic scattering or polarization analysis, considerably more. Thus, no neutron experinents have been per-formed with elements hear :er than curium and this seems unljkely to change within the next few years On the other hand, since neutrons easily penetrate most materials, once the actinide sanple is encapsulated in a vanadium, aluminum, or even steel container, then the experiment is no more difficult than on any other system.

\section{The Elements}

The extension of the wave functions in the elements leads to direct exchange effects that result in a number of complex crystallographic phases for each elerent and almost magnetic behavior for $\mathrm{U}, \mathrm{Np}$, and $\mathrm{Pu}$ [1]. For some time the idea that a spin-density wave occurred at $40 \mathrm{~K}$ in alpha-uranium was fashionable, but recently at Oak Ridge National Laboratory careful measurements of the phonon spectra [2] led to the discovery of a charge-density 
wave as responsible for the many anomalies at low tempcrature [3]. Since we are confining our attention to magnetic phenomena, we shall not describe this in detail, but the efforts do represent a major achievement for neutron scattering in view of the great nany studies undertaken on $\alpha-U$. The first element that exhibits spontaneous magnetic order is curium, as shown by Fournier et al. [4] by neutron diffraction. Unfortunately, this fixst experiment was not able to provide a solution to the antiferronagnetic structure of the metal, so that another experiment should be attomptcd.

\section{Localization of $5 f$ Electrons in Neptu: : $:$ Compounds}

Some years ago Hill [5] noticed that for many actinide compounds the occurrence of magnetic order could be correlated with a critical actinideactinide separation $\left(\mathrm{d}_{\mathrm{An}}\right)$. An excellent example of the validity of these ideas can be found in a serles of neptunium Laves phase compounds, which have been examined by magnetization, Mossbauer, and neutron techniques $[6,7]$. A relationship must be found between the hyperfine field, $\mathrm{H}_{\mathrm{hf}}$, measured by the Mossbauer technique, and the magnetic moment $\mu_{N}$ measured by neutron diffraction. Empirically a remarkably good linear relationship [8] exists between these two quantities and is shown in Fig. 1. This may be taken as strong evidence for localized $5 f$ electrons, and it is also significant that the best fit extrapolates through the theoretical values for $\mathrm{Np}^{3+}\left(5 f^{4}\right)$. However, as the moment becomes smaller we would not expect this relationship to continue to hold. A study of the compounds $\mathrm{NpAl}_{2}\left(\mathrm{~d}_{\mathrm{An}}=3.37 \AA\right.$ ) and. Npos $_{2}\left(d_{A n}=3.26 \AA\right)$ showed this very clearly [7]. The idea quite simply is that as the actinide ions are brought closer together in, for example, $\mathrm{NpRu}_{2}\left(\mathrm{~d}_{\mathrm{An}}=3.23 \stackrel{\circ}{\mathrm{A}}\right)$, the $5 f$ electrons bandwidth increases until it is too wide to support spontaneous ordering. In $\mathrm{NpOS}_{2}$ the moment is only $0.3 \mu_{3} / N_{P}$ 
atom and a comparison of the magnetization, Mossbauer, and neutron experiments led to the conclusion that it was an itinerant system with extended $5 f$ wave functions. Furtler confirmation of the itinerant nature of the magnetism in Npos 2 comes from specific heat measurcments [9].

$$
\text { Spin Lattice Interactions in } \mathrm{UO}_{2}
$$

Uranium dioxide was one of the first actinide materials studied in detail by neutron scattering. Frazer, et al. [10] diccovered the firstorder phase transition from the paramagnetic to antiferromagnetic state, but the electronic structure remained in some doubt until the calculations of Rahman and Runcinan [11] showed that the $\Gamma_{5}$ triplet of the predominant $3_{4}$ state was the ground state. Confimation of this came from infra-red spectroscopy [12] and neutron inelastic scattering [13]. Attempts to fit the magnon dispersion curves with a conventional exchange coupled two-sublattice model were only qurlitatively successful. The effect of introducing quadrupolequadrupole interactions [12] gave excellent agreement, so tiat in a system like $\mathrm{VO}_{2}$ with a large orbital ground-state degeneracy, the spin-lattice interaction leads to indirect quadrupole-quadrupole interactions that may be as strong as the exchange terms. One consequence of Allen's theory [12] was that the two oxygen sublattices should move with respect to each other. In 1974 we performed a careful neutron experiment at Argonne to measure the magnetic form factor and thereby attempt to identify the ground-state wave function (see below). To our surprise, a subset of the supposedly magnetic reflections appeared to get stronger with increasing $Q(=4 \pi \sin \theta / \lambda$, where $\theta$ is the Bragg angle and $\lambda$ the neutron wavelength). After a thorough analysis this 
subset of reflections was found to contain additional scattering from an internal rearrangenent mode involving the oxygen sublattices [14]. The oxygen atoms shift by $0.014 \AA$ from their special positions in the fluorite structure, sce Fig. 2. The uranium lattice remains undisturbed, that is why we call the deformation an "internal distortion". Allen [12] was almost right, except that it is a transverse optic zone boundary mode that condenses rather than the zone center one he proposed. The iricriguing question, however, is why $\mathrm{UO}_{2}$ chooses this particular deformation? Fig. 2 shows the effect, and also suggests a possible driving mechanism. The magnetic spins lie in the (001) plane and from the ground-state wave function we know that the magnetization density is oblate (i.e., it resembles a compresscd sphere with the quantization axis or dipole moment parallel to the compression axis); hence the quadrupolar electrostatic interactions are reduced with the spin configuration of Fig. 2. The spins are aligned parallel to the shortest diagonal of the parallelopiped formed by the oxygen atoms. This predicts a $2 \vec{q}$ (or 4 sublattice) magnetic structure for $\mathrm{UO}_{2}$, as shown in Fig. 2, rather than the single $\overrightarrow{\mathrm{q}}$ structure assumed heretofore, but without a single domain sample neutron diffraction is unable to differentiate between these two nodels [14].

The studies of $\mathrm{VO}_{2}$ with neutrons, both elastic [10,14] and inelastic [13], illustrate particularly well the power of this technique. Internal distortions, which were first predicted by Kanamori in 1960 [15], are important for our understanding of magnetoelastic interactions, and we can certainly expect thein to be found in other materials in which quadrupole moments are large. 
Actinide Compounds with the Rocksal.t Structure

The actinide compounds with the rocksalt structure have been the subject of many investigations and, since most of then order magnctically, neitrons have played an important role in elucidating theix properties. Tabulations of: their various properties have appeared a number of times $[1,16,17]$ and wil1 not be repeated here. To a large extent their magnetic properties may be understood by assuming local moments, although th. a high values for the low-temperature specific heat coefficients $\gamma\left(50 \mathrm{~mJ} / \mathrm{mole} x \mathrm{k}^{2}\right.$ for $\mathrm{WN}[16]$, for example) suggests a ligh density of $5 f$, and possibly also $6 d$, states at the Fermi level. What is not understood is the nature of the ground-state wave functions and the coupling between the moments, and four classes of neutron experiments will be described to illustrate some recent developments.

\section{1) Magnetic Structures}

Neutron diffraction has historically been the most important technique for determining the arrargement of moments in materials and has been applied to a great number of actinide systems. Among those compounds with the NaC1 crystal structure the UX (X $=S$, Se, and $\mathrm{Te}$ of group VIa) are ferromagnets whereas the UX ( $X=N, P, A S, S b$, and $B i$ of group Va) are simple antiferromagnets.

However, when these compounds are mixed to form pseudo-binaries, e.g., ${ }^{U A s}{ }_{1-x} S_{x}$, then the magnetic structures become very complex, with the magnetic and crystallographic unit cells becoming incommensurate for certain compositions [18]. Long periodic structures are also found in the neptumium binary compounds and some of these are illustrated in Fig. 3 [19]. Despite the apparent complexity of these structures, there is a remarkable unifying feature, wh:.2h way be stated as follows: The spin arrangements consist of ferromagnetic (001) 
planes with the spin direction perpendicular to the plane, then these planes are stacked in different ways such that the $\vec{\tau}$ vector, where the repeat distance in real space is $1 / \tau$, may take on a variety of values. Arrangenents such as $5+, 4-$ and $4+, 3-$ have been reported [18]. Such a recurring theme within the structures of all antiferromagnetic uranium and neptunium compounds led to the simple concept that perhaps the coupling within the ferromagnetic planes was considerabl: stronger than the (presumably indirect) exchange between the planes.

\section{2) Long-range Magnetic Correlations}

When single crystals became available one of the first experiments was to search for the directional anisotropy inferred in the previous section. This is best done by studying the long-range magnetic correlations just before the material orders magnetically (i.e., in the critical regime). The crystal chosen was USb, which orders with the simple type I structure shown in Fig. 3 [20]. To visualize the experimental method we have drawn the [1̄0] projection of the reciprocal lattice in the upper half of Fig. 4. Brags points from the fcc atomic structure are (000) and (1.11). In principle, both (001) and (1.10) are magnetic points arising from magnetic domains with a [001] propagation direction. However, no Bragg peak occurs at (001) because the spin direction is then parallel to the scattering vector. Around the (001) point we should observe transverse fluctuations of the spin system in the critical region. The result of a scan from $C$ to $C^{\prime}$ at $T_{N}+3 K$ is shown in the lower part of Fig. 4. No critical scattering has been observed around the (001) point, or equivalent (100) and (010) points, at any temperature. Quantitatively we can say that $\chi_{T}<0.01 \chi_{L}$, where $\chi_{T}$ is the transverse susceptibility and $x_{L}$, the 
longitudinal susceptibility. This is an important result, with two immediate consequences: (1) The anisotropy is considerable - one could even argue that it defines an Ising system. (2) The critical scattering at the (110) position represents the longitudinal susceptibility directly.

At the (110) position we can perform scans in two directions. These are shown as $A A^{\prime}$ and $B B^{\prime}$ in the upper part of Fig. 4. The actual scans at $\left(\mathrm{T}_{\mathrm{N}}+3 \mathrm{~K}\right)$ are shown in the lower part of Fig. 4. The critical scattering is by no means isotropic around the (11.0) point, but shows a very diffuse nature along the direction parallel to the spin direction. Such behavior is very reminiscent of two-dinensional systems, such as $\mathrm{K}_{2} \mathrm{NiF}$, [21] in which the scattering near $\mathrm{T}_{\mathrm{N}}$ appears in the form of rods of jntensity. However, this behavior has not been observed in cubic materials. Similar results have been found in experiments on UN [22] but efforts to make measurements with the ferromagnets are difficult since with $\vec{\tau}=0$ the scattering from all domains is superintposed in reciprocal space, thus averaging any microscopic anisotropy [23].

\section{3) Magnetic Form Factors}

At first glance a neutron magnetic form factor measurement would appear to be the ideal way to resolve the question of whether the electrons are localized or itinerant, since one sees directly the spatial extent of the unpaired (i.e., magnetic) electrons. Experiments on the transition metals and compounds have shown that this is too simple an interpretati as the spin density of the wave functions near $E_{F}$ is often very much like that of a free aton. In extreme cases, and perhaps $\mathrm{NpOs}_{2}$ [7] is such an example, the wave functions are really delocalized, but very often a more interpretable measurement 
is whether or not the form factor exhibits asphericity, in which case a deduction may often be made about the ground-state wave function.

The first experiments on US by Wedgwood [24] illustrated the difficusty in analyzing data that was essentially spherically symmetric, and we have found the same kind of form factor with recent work on the ferromagnets UTe and $\mathrm{USb}_{0.8^{\mathrm{Te}}} 0.2$ [25]. In contrast, the form factor of USb [26] showed considerable anisotropy. A detailed analysis in terms of possible crysta1ficld states led to the assignment of a $5 f^{3}$ configuration with a wave function dominated by the $M=17 / 2\rangle$ component. Our expectation, based on crystal-field arguments as well as the analogous lanthanide compound $\mathrm{NdSb}$ [27], had been for a ground state vave function consisting primarily of the $|M=9 / 2\rangle$ component, which has a prolate distribution of magnelization density about the ordered moment. Instead, in USb the magnetization density is definitely oblate in shope, and we show this schematically in Fig. 5. Here we also give a conceptual picture of the $f-p$ hybridization that has been advanced [28] to understand the nature of the ground-state wave functions and why the very large difference occurs between the interactions within the ordered (001.) planes and between then, as discussed in the previous section. However, the more quantitative predictions of this idea have still to emerge.

In uranium compounds the free-atom form factors of the $5 f^{2}$ and $5 f^{3}$ configurations look almost identical [29], so that to obtain useful information we have concentrated on the aspherical features, where they exist. For transuranium systems, on the other hand, the genera: shape of the form factor may be able to give unambiguous information. This is particularly true in the case of plutonium in its trivalent state, In which case (as with $\mathrm{Sm}^{3+}$ ) $\vec{J}=\vec{L}-\vec{S}$ 
and $\overrightarrow{\mathrm{L}}(=5)$ and $\vec{S}(=5 / 2)$ partially cancel. In Fig. 6 we show the magnetic form factor as measured in two ferromagnets Pup and PuFe 2 [30], both measurements were performed with polarized neutrons on polycrystalline samples. The remarkable flatness of the form factor out to high scattering angles is because the total magnetization density has a negative region in real space. Such an unusual form factor allows us immediately to assign the trivalent ionization state to these compounds, and in the case of PuP we were also able to give an upper limit to the extent of $J$ mixing. This was considerabiy less than previo:sly thought, making the neutron experinent doubly useful. 4) Crystal-field Levels and Collective Excitations

The measurement of the elementary magnetic excitations by neutron inelastic scattering provides detailed information about the ground and excited state wave functions and the nature, strength, and possible anisotropy of the exchange interactions. On metallic actinide systems the first experinents were performed on polycrystaline UX samples by Wedgwood [31]. In view of the importance of the crystal-field interactions it was therefore surprising that no sign of any discrete crystal-field levels were found with neutron spectroscopy. The investigations on polycrystalline samples should be able to see dispersionless exciton levels, but cannot hope to measure collective excitations, which are at discrete position in $\vec{Q}, w$ space. For these studies large $(\geq 2 \mathrm{~g}$ ) single crystals are needed. The first experiments on UN, however, provided even greater mysteries since no discrete excitations at all were seen [22]. More recently, poorly defined excitations have been seen in un near the magnetic zone center, and this work is continuing at Clalk River with the first experiments on ferromagnet UTe [32]. 
A rather detailed examination of the collective excitations has now been performed at the Institut Laue Langevin on USb and we reproduce the dispersion curves in Fig. 7[33]. The most surprising features are: (a) The observation of a longitudinally polarized mode at low frequency, i.e., a modulation of the size of the magnetic moment along the quantization axis, rather than a conventional spin wave, which corresponds to the precession of the magnetic moments about the quantization axis. (b) At the magnetic zone center, $X$ point (1.10), the collective magnetic excitation has the same frequency as the phonon. (c) As the temperature is raised the amp.litude of this collective excitation rapidly decreases and is essentially unobservable above $T_{i \mathrm{~N}} / 2$. Further details of these rather long and complex experjunents are beyond the scope of the present article; suffice it to say that models hased on lanthanide type of behavior are quite unable to account for these results, so we have a very exciting situation where we can anticipate both new experiments and (hopefully) greater attention from the theorists.

\section{Discussion}

No mention has been made in this article of neutron methods, formulae, or technology. As one would expect, the techniques developed for other elements and compounds of the periodic table have proved their worth in actinide investigations, and the references contain full details. Our aim has been to address the question of what makes the actinides so interesting from the viewpoint of a solid-state physicist, and why neutron scattering is such an important tocl in these studics. The special advantages of neutron scattering include: (a) The penetrating power of the neutron, thus making encapsulation relatively easy for active samples. (b) The sensitivity 
to light atoms in the presence of heavy ones, and cramples range from the early identification that: UN had the rocksalt structure [34] to the measurement of the lattice dynanics [35] and the internal distorion [14] in $60_{2}$. (c) From the interaction of the neutron with the magnetic moments we are able to deduce magnetic structures, the value of the localized magnetic moment, the shape of the $5 f$ electron wave functions, and the extent of the magnetic correlations. We can also use the extreme sensitivity of the polarizotreutron technique to measure the induced magnetic noment (as smal.1 as $0.011_{B}$ ) in paramagnetic sysiens, and determine the spatial extent of this induced naghetjzation throughout the unit cell [36]. These measurements are of particular interest since they can be compared with theoretical spin densities, and thus form the basis for a rigorous test of the eigenfunctions used in fitting the de Haas van Alphen frequencies [37]. (d) From the fact that neutrons have energies close to those of elementary excitations $(0.01-0.1 \mathrm{eV})$ we are able to measure phonons [35] and study the dynanics of phase transitions [3]. Combining the magnetic interaction with jnelastic scattering we can search for crystal-field levels, measure the collective magnetic excitations, and the generalized magnetic response function $S(\vec{Q}, \omega)$. This latter measurement tells us something abou rine spin dynamics and whether such materials as $\mathrm{UAl}_{2}$ can be assigned a distu.lct $5 f$ electron configuration [38].

The dominance of majizetic interactions is for a good reason. It is the unpaired $5 f$ electrons that give the actinides and their compounds such a variety of properties, and by utilizing the magnetic intcraction we have a probe that selects out the very unpaired electrons of greatest interest. 
There are additional areas that are now being explored, but which space does not permit us to discuss, e.g., a search for a possible internal distortion in $\mathrm{N}_{2} \mathrm{O}_{2}$, a search for paramagnon fluctuations in $\mathrm{UAl}_{2}$, uniaxial. stress measurements on UN and UAs, high field studies (>90 kOe) of the magnetic phase transitions in UAs, the study of complex magnetic structures with single crystals, and polarization analysis experiments on PuP to search For the conductionelectron polarization.

The grcatest dicadvantage of neutron scattering is that the fluxes are low and the interactions are weak. We therefore often need large samples. Within the foreseeable future we cannot expect much respite from this requirement, especially as we turn to more and more complex neutron technology to solve the physics. However, the ability to produce single crystals of uranium compounds augurs well for the future of this field, since the chemistry and metallurgy of the first few actinide elements and their compounds are not substantially different. To obtain single crystals of transuranim compounds, and thus realize the full potential of neutron scattering, presents great difficulties, but a rich harvest certainly awaits those Laboratories willing to accept the challenge.

\section{Acknowledgments}

The production of single crystals of uranium compounds has proved the key to many of the new developments discussed above, and I am particularly indebted to Oscar Vogt of ETI, Zurich for the major role he has played in this field. I should like to record my thanks for collaboration and discussions with A. J. Arko, P. J. Brown, W. J. L. Buyers, B. R. Cooper, A. Delapalme,
A. J. Freeman, T. M. Holden,
J. F. Reddy, J. Rossat-Mignod, S. K. Sinha,
W. G. Stirling and H. G. Smith. 


\section{References}

* Work supported by the U. S. Bepartment of Energy.

[1] "The Actinides: Electronic Structure and Related Properties",

A. J. Freeman and J. B. Darby, ed. (Acadenic Press, N.Y. 1974) vol. I and IJ.

M. B. Brodsky, Rep. Progr. Physics. 41 (1978) 1547.

[2] W. P. Crumett, H. G. Smith, R. M. Nicklow, and N. Wakabayashi, Phys. Rev. B (in press).

[3] H. G. Smith, N. Wakabayashi, W. P. Crumett, R. M. Nicklov, G. H. Lander and E. S. Fisher, to be published in AIP Conf. Proc. as Proceedings of Conference on modulated structures, Hawaij, March, 1979.

[4] J. M. Fournier, A. Blaise, W. Muller and J. C. Spirlet, Physica 86-85B (1977) 30.

[5] H. liill, in "Plutoniuin and other Actinides," W. N. Miner, ed. (AIHE, New York, 1971) p. 2.

[6] A. T. Aldred, B. D. Dunlap, D. J. Lam and I. Nowik, Phys. Rev. B 10 (1974) $1011 ;$ B 11 (1975) 530 .

[7] A. T. Aldred, B. D. Dunlap and G. H. Lander, Phys. Rev. B 14 (1976) 1276.

[8] B. D. Dunlap and G. H. Lander, Phys. Rev. Letters 33 (1974) 1046

[9] M. B. Brodsky and R. J. Trainor, Physica 91B (1977) 271.

[10] B. C. Frazer, G. Shirane, D. E. Cox and C. E. O1sen, Phys. Rev. 140 (1965) A1448.

[11] H. U. Rahman and W. A. Runcíman, J. Phys. Chem. Solids 27 (1966) 1833;

30 (1969) 2497; H. U. Rahnan, Physica 45 (1970) 511.

[12] S, J. Allen, Phys, Rev. 166 (1968) 530; 167 (1968) 492.

[13] R. A. Cowley and G. Dolling, Phys, Rev. 167 (1968) 464; Phys. Rev. Letters 16 (1966) 683 . 
[14] J. Faber and G. H. Lander,Phys. Rev. B 14 (1976) 1151; Phys. Rev. Letters 35 (1975) 1770 .

[15] J. Kanamori, J. Appl. Physics 31 (1960) 14S.

[16] J. Grunzweig-Genossar, M. Kuznietz and F. Friednan, Phys. Rev. 173 (1968) 562 .

[17] G. H. Lander, Inst. Physics (London) Conf. Series $\underline{37}$ (1978) 173.

[18] H. Kuznietz, G. H. Lander and Y. Baskin, J. Appl. Physics 40 (1969) 1130; Phys. Rev. 188 (1969) 963; J. Leciejewicz, A. Murasik, R. Troc, and T. Palewski, Phys. Stat. Solidi 46 (1971) 391; G. H. Lander, M. H. Kueller and J. F. Reddy, Phys. Rev. B $\underline{6}$ (1.972) 1880.

[19] A. T. Aldred, B. D. Dunlap, A. R. Harvey, D. J. Lam, G. H. Lander and M. H. Mueller, Phys. Rev. B 9 (1974) 3766.

[20] G. II. Lander, S. K. Sinha, D. M. Sparlin and O. Vogt, Phys. Rev. Letters $\underline{40}$ (1978) 523 .

[21] R. J. Birgeneau, J. Skalyo and G. Shirane, Phys. Rev. B 3 (19;1) 1736; J. Appl. Phys. 41 (1970) 1303.

[22] W. J. L. Buyers, T. M. Holden, E. C. Svensson and G. H. Lander in "Proc. of Int. Symposium on Neutron Inelastic Scattering (Int. Atomic Energy Agency, Vienna, 1978) p. 239.

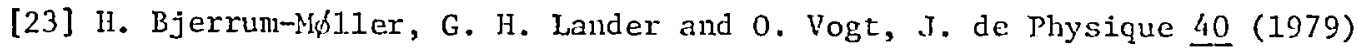
C4-28.

[24] F. A. Wedgwood, J. Phys. C $\underline{5}$ (1972) 2427.

[25] G. Busch, O. Vogt, A. Delapalme and G. H. Lander, J. Phys. C 12 (1979) 1391.

[26] G. H. Lander, M. H. Mueller, D. M. Sparlin and O. Vogt, Phys. Rev. B 14 . (1976) 5035. 
[27] A. Furrer, W. J. L. Buyers, R. M. Nicklow and O. Vogt, Phys. Rev. B 14 (1976) 179.

[28] S. K. Sinha and A. J. Fedro, J. de Physique 40 (1979) C4-214.

[29] A. J. Freeman, J. P. Desclaux, G. H. Lander and J. Faber, Phy's. Rev. B I3 (1976) 1168; J. P. Desclaux and A. J. Frecnan, J. Magn. \& Mag. Matls. 8 (1.978) 119 .

[30] PuP: G. H. Lander and D. J. Lam, Phys. Rov. B 14 (1976) 4064

PuFe $_{2}$ : G. H. Lander, A. T. Aldred, B. D. Dunlap and G. K. Shenoy, Physica 86-88B (1977) .152 .

[31] F. A. Wedgrood, J. Phys. C I (1974) 3203.

[32] W. J. L. Buyers, A. F. Nurray, E. C. Svensson, T. M. Holden, P. du Plessis, G. H. Lander and 0 . Vogt, submitted to Int. Conf. on "Neutron Scattering and Magnetism", Julich, August 1979, to be published in J. Mag. \& Mag. Mat]s.

[33] G. H. Lander, W. G. Stirling and O. Vogt, Phys. Rev. Letters 42 (1979) 260; Phys. Rev. (to be published).

[34] M. H. Mueller and H. W. Knott, Acta Crystallogr. 11 (1958) 751.

[35] G. Dolling, A. D. B. Woods and R. A. Cowley, Can. J. Phys. 43 (1965) 1397.

[36] $\underline{U R h}_{3}$ : A. Delapalme, G. H. Lander and P. J. Brown, J. Phys. C 11 (1978) 1441;

$\underline{\alpha-U}:$ R. C. Maglic, G. H. Lander, M. H. Mueller and R. Kleb, Phys. Rev. B 17 (1.978) 308;

$\mathrm{UGe}_{3}$ : G. H. Lander, J. F. Reddy, A. Delapalme and P. J. Brown, see Proceedings as in Ref. [32].

[37] $\underline{\mathrm{URh}}_{3}$ : A. J. Arko, H. B. Brodsky, G. W. Crabtree, D. Karim, D. D. Koelling, L. R. Windmiller and J. B. Ketterson, Phy's. Rev. B 12 (1975) 4102. $\underline{\text { UGe }}_{3}:$ A. J. Arko and D. D. Koelling, Phys. Rev. B 17 (1978) 3104. [38] M. Loewenhaupt, S. Horn, F. Steglich, E. Holland-Moritz and G. H. Lander, J. de Physique 40 (1979) C4-12. 


\section{Pigure Captions}

Fig. 1. Linear relationship between the hyperfine field $\mathrm{H}_{\text {hf }}$ and the magnetic monent $\mu_{\mathrm{N}}$ in neptunium intermetallics. Experimental. points are shown by closed circles and calculated free-ion values by open circles [8]. MSD Neg. No. 61887 .

Fig. 2. (OOI) projection of the fluorite structure. The closed and open circles represent uranium atoms at $z=0$ and $z=1 / 2$, respectively. The large circles represent oxygen atoms at $z=1 / 4$ and $z=3 / 4$ displaced from the ideal fluorite lattice (indicated by the dashed Iines). The shift of the oxygen atoms is not dram to scale, $\Delta / a=2.6 \times 10^{-3}$. The suggested noncollinear spin confjguration is also shown. MSD Neg. No. 61835.

Fig. 3. Magnetic structures of $\mathrm{NpX}$ compounds [19]. NpN is a ferromagnet with a <111> easy axis. NPP has a sinusoidal modulation of the magnetic moment along the propagation axis with a repeat of 3 unit cells, 6 (001) planes. At high temperature, NpAs has a 4+, 4-, structure but has a first-order transition at $142 \mathrm{~K}$ to the type-I, +-, structure, which is also the structure of $\mathrm{NpSb}$. ANL Neg. No.

Fig. 4. Measurement of long-range magnetic correlations in USb [20]. Upper section, [1]̄o] projection of the reciprocal lattice. Lower section, experimental points and least-squares fits (solid lines) for the scans as shown in the upper section. The small bars indicate the experimental. resolution functions. MSD Neg. Nos. 62919 and 64680 . 
Fig. 5. This shows schenatically the oblate magnetization density [26] at the uranium sites (solid circles) and the proposed overlap of the anion (open circles) $p$ waverunctions [28]. Notice that because the $5 f$ wave function is extenced in the (001) plane the overlap is much stronger in this plane than between the planes.

Tig. 6. Magnetic form factors of $\mathrm{pu}^{3+}$. Upper curve, results for PuP [30]. The solid and dashed curves are the best fits to the data with $5 f^{5}$ and $5 f^{4}$ confichurations, respectively. The arrow on the ordinate axis gives the total moment determined by magnetization experinents, the discrepanfy of $\sim 0.3 \mu_{B}$ between this and the neutron experiment being due to conduction-electron polarization. The insert shows the relativi:ptic Dirac-Fock values for $\left\langle j_{0}\right\rangle$ and $\left\langle j_{2}\right\rangle$, see Ref. [29]. The lower figures are form factors measured in $\mathrm{PuFe}_{2}[30]$. Notice that, althoygh the $\mathrm{Pu}$ moment is $n 0.4 \mu_{\mathrm{B}}$ as compared to $1.4 \mu_{\mathrm{B}}$ for the iron moment, at high angles the scattering is dominated by the $\mathrm{Pu}$ froment because of the unusual $\mathrm{Pu}^{3+}$ form factor.

Fig. 7. The dispersion curves for USb [33]; energy plotted against wavevector transfer $\vec{Q}$ (in units of $2 \pi / a$ ). The dashed lines represent the phonons with the open points as measurements. The solid points are the longitudinally polaxized collective excitation with a $1.5 \mathrm{THz}$ $(=6.2 \mathrm{meV})$ anisotropy gap at the zone center (X point). A dispersionless (and temperature independent) exciton, which is probably a crysta]-field level, is located at $26.5 \mathrm{THz}(=27 \mathrm{meV})$ and indicated by the hatched area. 


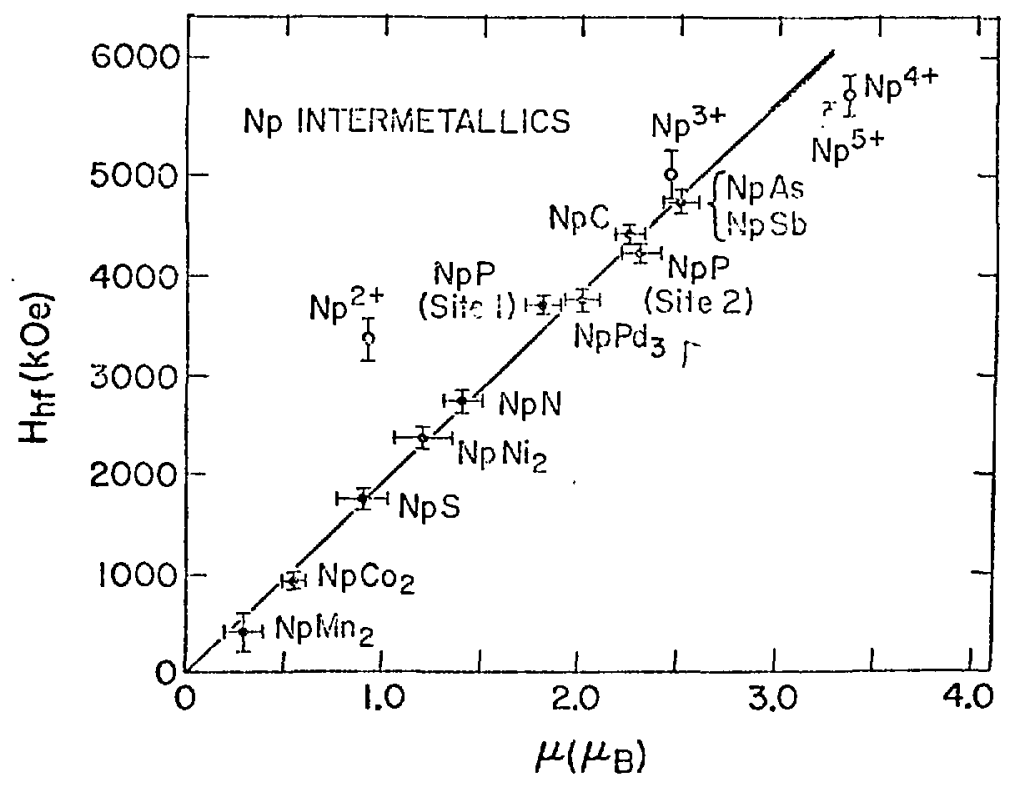

Fig. 1. Linear relationship between the hyperfine field $\mathrm{H}_{\mathrm{hf}}$ and the magnetic moment $\mu_{N}$ in noptunium intermetallics. Experimental points are shown by closed circles and calculated free-ion values by open circles. [8] MSD Neg. No. 61887 


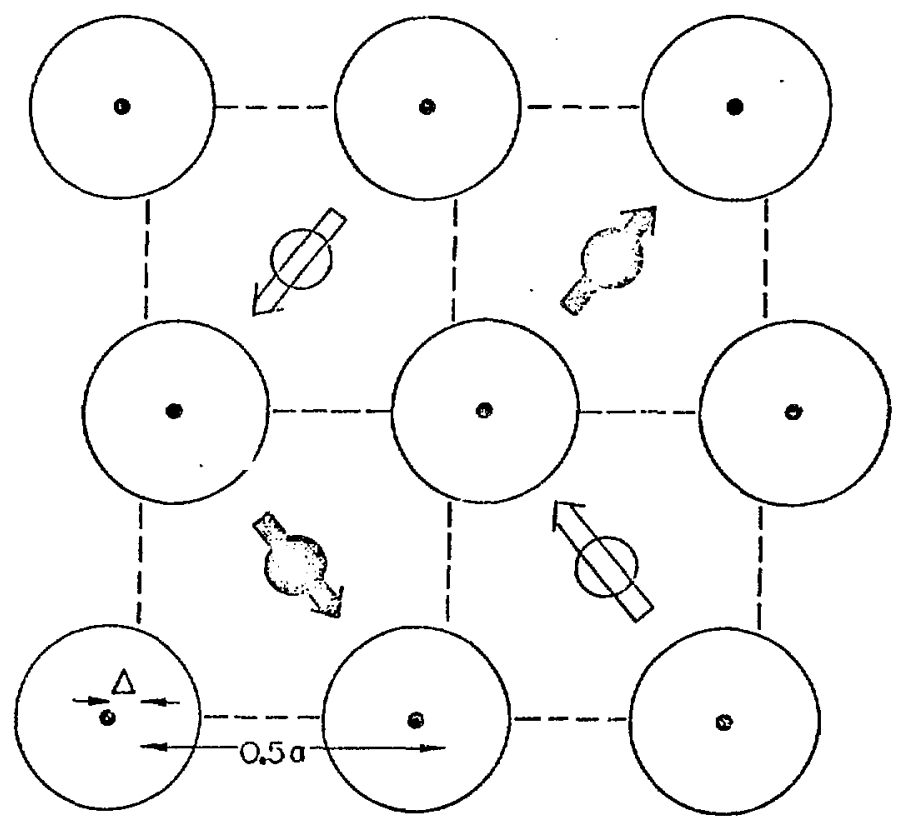

Fig. 2. (001) projection of the fluorite structure. The closed and open circles represent uraniun atoms at $z=0$ and $z=1 / 2$, respectively. The large circles ropresent oxygen atoms at $z=1 / 4$ and $z=3 / 4$ displaced from the ideal fluorite latice (indicated by the dashed lines). The shift of the oxygen atoms is not drawn to scale, $\Delta / a=2.6 \times 10^{-3}$. The suggested noncollinear spin configuration is also shown. MSD Neg. No. 61835. 


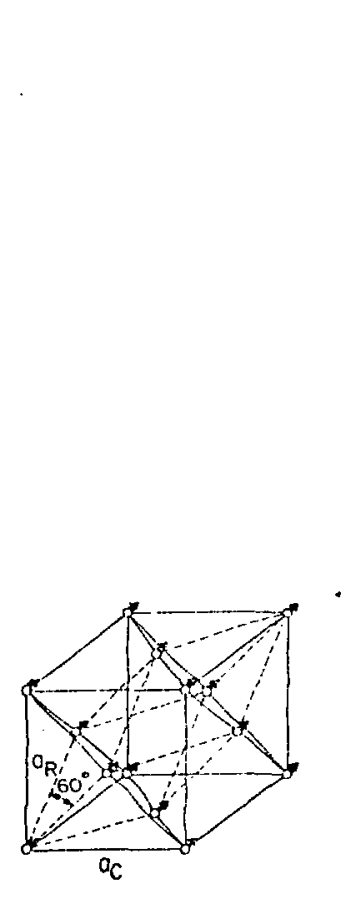

$N p N$

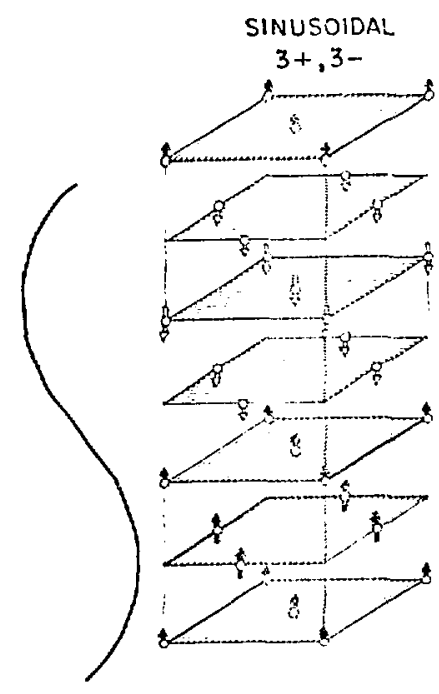

NpP
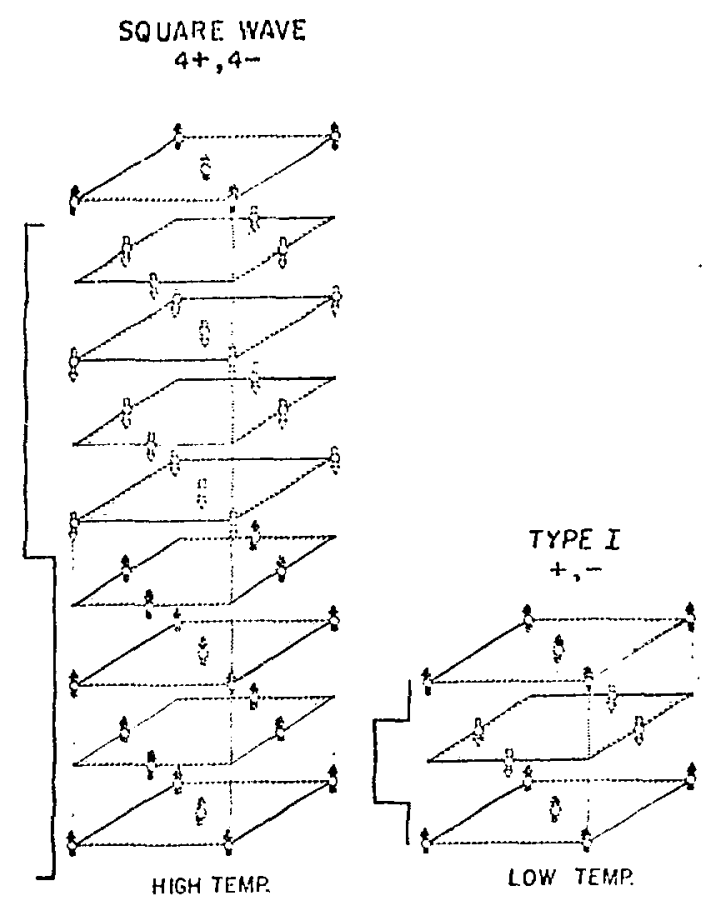

Np As

Fig. 3. Magnetic structures of NpX compounds [19]. NpNi is a ferromagnet with a <111> easy axis. NpP has a sinusoidal modulation of the magnetic moinent along the propagation axis with a repeat of 3 unit cells, 6 (001) planes. At high temperature, NpAs has a 4+, 4-, structure but has a first-order transition at $142 \mathrm{~K}$ to the type-I, +-, structure, which is also the structure of $\mathrm{NpSb}$. 

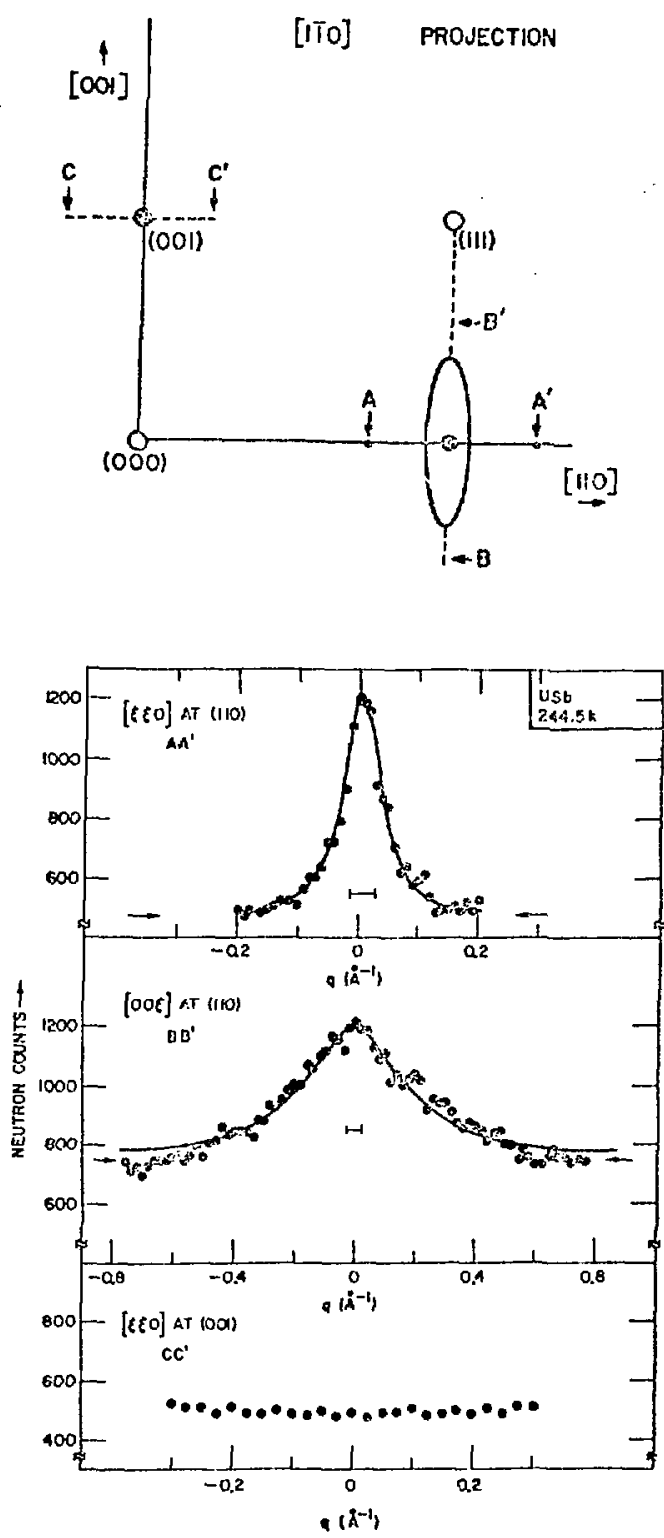

Fig. 4. Measurenent of long-range magnetic correlations in USb [20]. Upper section, [1]0] projection of the reciprocal lattice. Lower section, experimental points and least-squares fits (solid lines) for the scans as shown in the upper section. The small bars indicate the experimental resolution functions. MSD Neg. Nos. 62919 and 64680. 


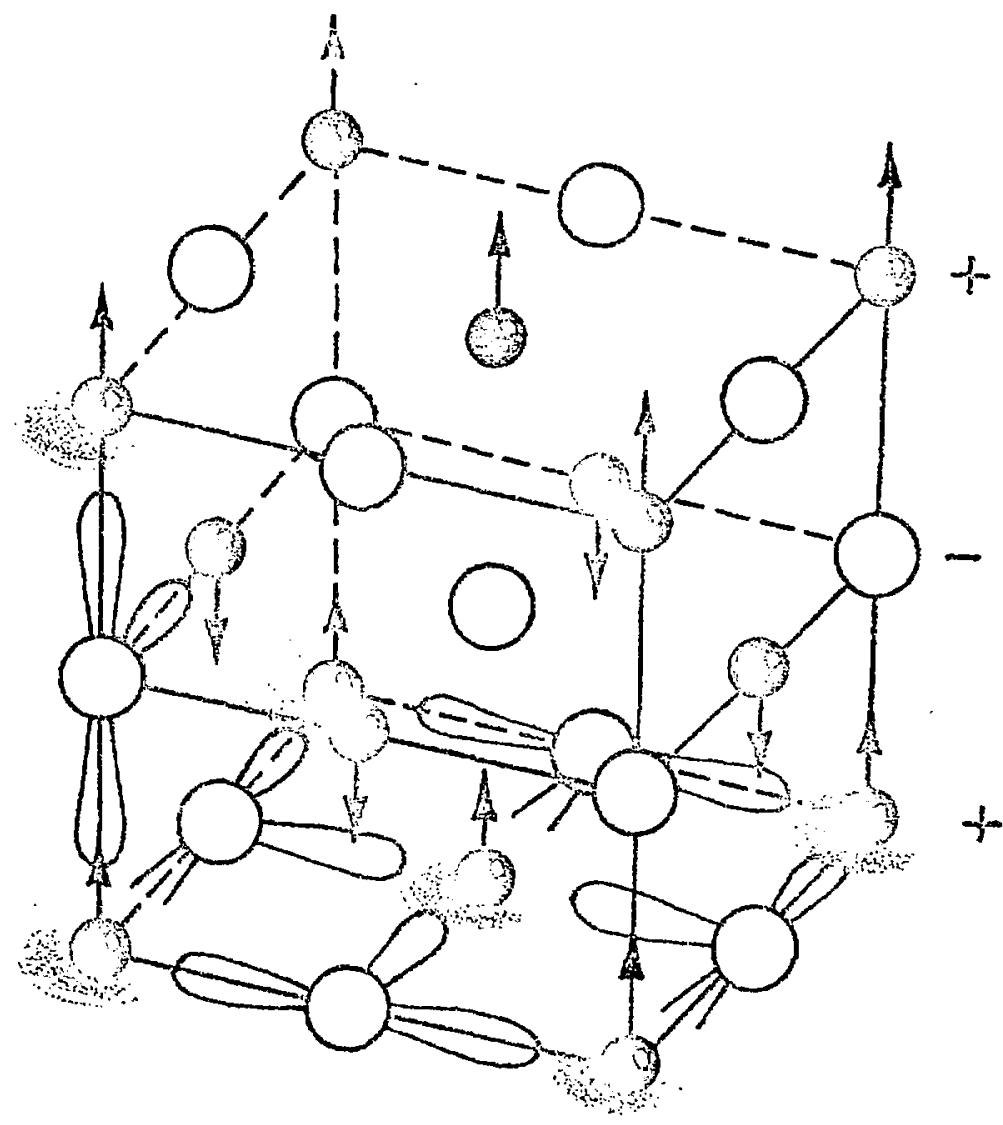

Fig. 5. This shows schenatically the oblate manetization density [26] at the uranium sites (solid circles) and the proposed overlap of the anion (open circles) $p$ wavefunctions [28]. Notice that because the $5 f$ wave function is extended in the (001) plane the overlap Is much stronger in this plane than between the planes. 
Figure 6.

Wagnetic form factors of $\mathrm{Pu}^{3+}$. Upper curve, results for pup [30]. The solid and dashed curves are the best its to the data with $5 f^{4}$ and $5 f^{4}$ configurations, respectively. The arrow on the ordinate axis gives the total. moment deternined by magnetization experiments, the discrepancy of $n 0.3 \mu_{B}$ between this and the neutron experiment being due to conciuction-clectron polarization. The insert shows the relativistic Dirac-Fonk values for $\left.<j_{0}\right\rangle$ and $\left\langle j_{2} s\right.$, see Ref. [29]. The lower figures are form factors measured in PuFe 2 [30]. Notice that, al.though the pu moment is $0.4 \mu_{B}$ as compared to $1.4 \mu_{B}$ for the ixon monent, at high angles the scattering is dominated by the Pu moment because of the unusual $\mathrm{Pu}^{3+}$ form factor.
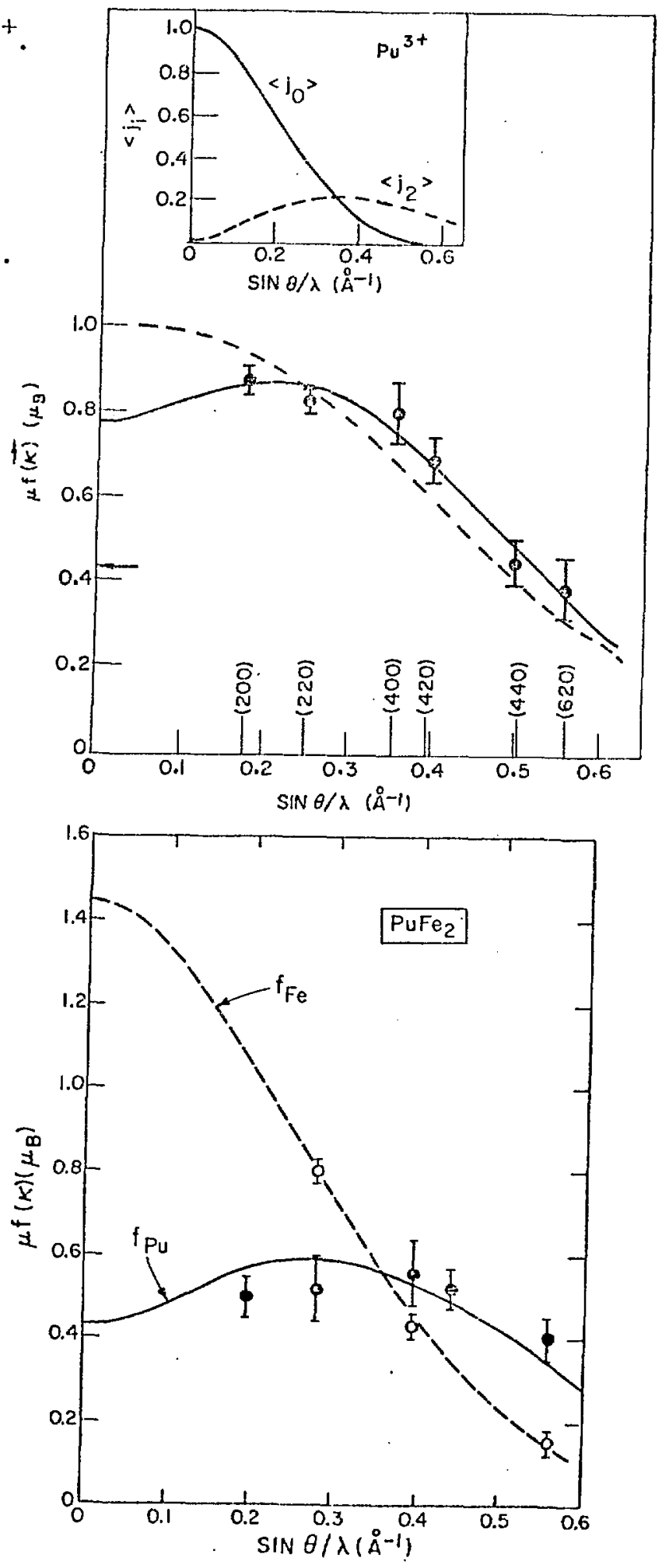


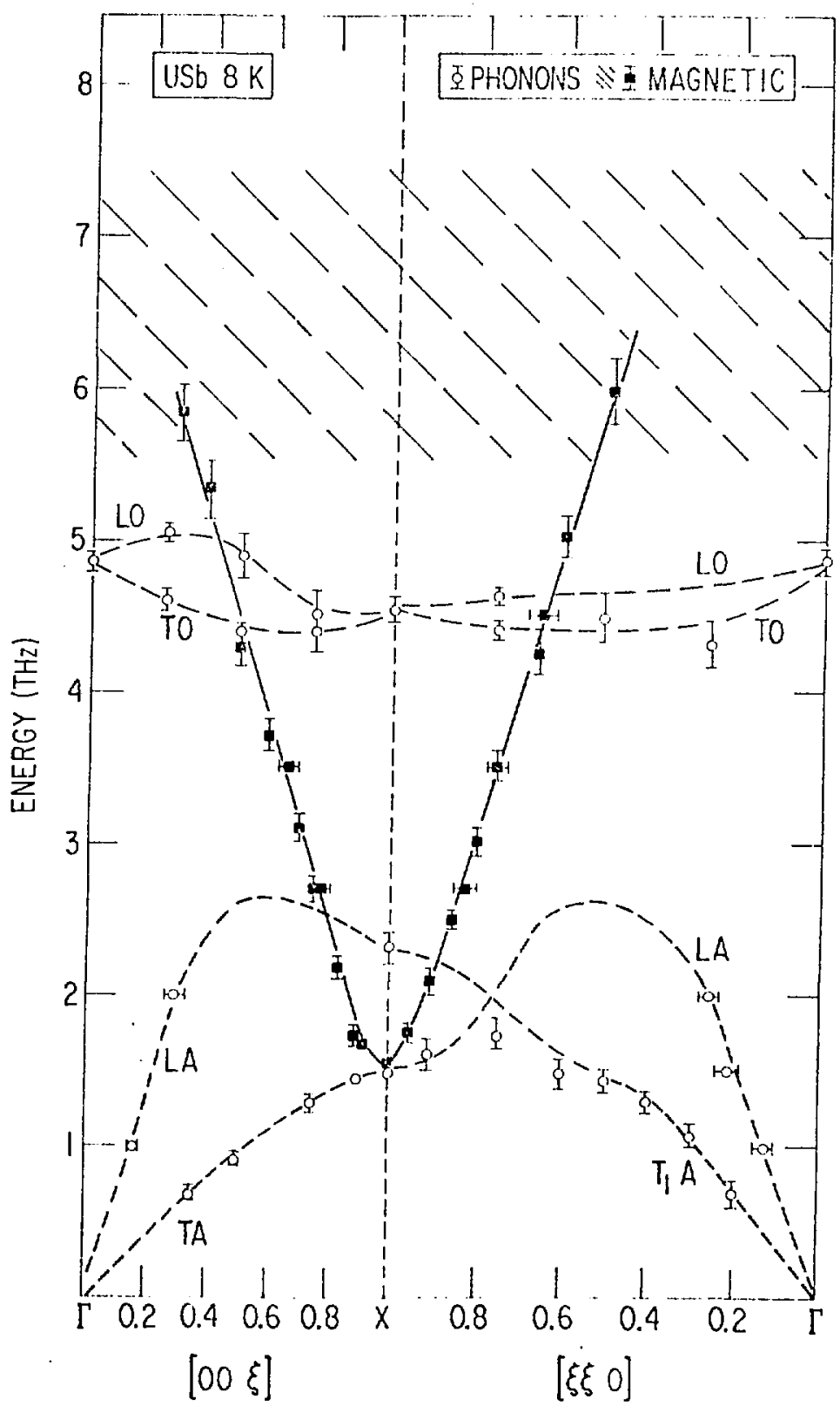

Fig. 7. The dispersion curves for USb [33]; energy plotted against wave-vector transfer $Q$ (in units of $2 \pi / a$ ). 'lhe dashed lines represent the phonons with the open points as measuroments. The solid points are the longitudinally polarized collective cxcitation with a $1.5 \mathrm{THz}(=6.2 \mathrm{mov})$ anisotropy gap at the zone conter ( $X$ point). $\Lambda$ dispersionless (and temperature independent) exciton, which is probably a crystal-ficld level, is located at $06.5 \mathrm{THz}(+27 \mathrm{meV})$ and findicated by the hatched area. 